\title{
ON THE BEHAVIOR OF QUASIREGULAR MAPPINGS IN THE NEIGHBORHOOD OF AN ISOLATED SINGULARITY
}

\author{
Pentti Järvi
}

1. The purpose of the present study is to consider the behavior of quasimeromorphic mappings about an essential isolated singularity or, by contraposition, to seek conditions under which such maps possess a limit at a singular point. In Section 2 we will establish two Picard-type theorems for quasiregular mappings both of which generalize a recent result of Vuorinen [14]. Furthermore, we will show that isolated singularities are removable for normal quasiregular mappings. Here normality means uniform continuity with respect to the quasihyperbolic metric. Section 3 is devoted to the value distribution of normal quasimeromorphic mappings. In Section 4 we will discuss the oscillation of quasimeromorphic mappings in the neighborhood of an essential singularity. This part of the work is related to Lehto's result concerning the growth of the spherical derivative of meromorphic functions near an isolated singularity [5].

I wish to thank Matti Vuorinen for his useful comments.

2. Our notation and terminology will be mainly the same as in Vuorinen's book [13]. Accordingly, $B^{n}(x, r)$ denotes the ball centered at $x \in \mathbf{R}^{n}$ with radius $r$ while $S^{n-1}(x, r)$ is the sphere with the same center and radius. For brevity, $B^{n}(r)=B^{n}(0, r), B^{n}=B^{n}(1), S^{n-1}(r)=S^{n-1}(0, r), S^{n-1}=S^{n-1}(1)$. The euclidean distance in $\mathbf{R}^{n}$ is denoted by $d(\cdot, \cdot)$, whereas $q(\cdot, \cdot)$ refers to the chordal distance in $\overline{\mathbf{R}}^{n}$, the one-point compactification of $\mathbf{R}^{n}$, which as usual is identified with the Riemann sphere $S^{n}\left(\frac{1}{2} e_{n+1}, \frac{1}{2}\right)$ (see [13, p. 4]). Let $D$ be a proper subdomain of $\mathbf{R}^{n}$. Then the quasihyperbolic distance $k_{D}(\cdot, \cdot)$, or briefly $k(\cdot, \cdot)$, is defined to be the metric induced by the density $d(x, \partial D)^{-1}$, where $d(x, \partial D)$ stands for the distance of $x$ and $\partial D$, i.e., $d(x, \partial D)=\inf \{d(x, y) \mid y \in \partial D\}$; see $[13$, p. 33]. Because nonconstant quasimeromorphic mappings are open, we may refer to the maximum and minimum principles in the same way as in planar function theory.

Some results of this section rely heavily on the following generalization of Picard's theorem due to Rickman [11]: For every integer $n \geq 2$ and each $K \geq 1$ there exists a positive integer $p=p(n, K)$ such that if $f: \mathbf{R}^{n} \rightarrow \mathbf{R}^{n} \backslash\left\{a_{1}, \ldots, a_{p-1}\right\}$ is $K$-quasiregular and $a_{1}, \ldots, a_{p-1}$ are distinct points in $\mathbf{R}^{n}$, then $f$ is constant. 
As in classical function theory Montel's theorem can be derived from Picard's theorem, so Rickman's result entails the following generalized Montel's theorem [9, Theorem 4]: Let $\mathcal{F}$ be a family of $K$-quasimeromorphic mappings in a domain $D \subset \mathbf{R}^{n}, n \geq 2$. If $A=\left\{a_{1}, \ldots, a_{p}\right\},(p=p(n, K))$ is a set of different points in $\overline{\mathbf{R}}^{n}$ such that $A \cap f D=\emptyset$ for all $f \in \mathcal{F}$, then $\mathcal{F}$ is a normal family.

In what follows $p(n, K)$ always refers to the number mentioned above.

Theorem 1. Let $R>0$ and let $f: \mathbf{R}^{n} \backslash \bar{B}^{n}(R) \rightarrow \mathbf{R}^{n}$ be a $K$-quasiregular mapping. Let $E=\left\{a_{j} \mid j \in N\right\}$ be a subset of $\mathbf{R}^{n} \backslash \bar{B}^{n}(R)$ such that

(1) there is an increasing sequence $\left(r_{j}\right)$ of positive numbers $\left(r_{1}>R\right)$ tending to $\infty$ and a constant $s>1$ such that for $A_{j}=\left\{x \in \mathbf{R}^{n}\left|r_{j}<\right| x \mid<s r_{j}\right\}$, $A_{j} \cap E=\emptyset$ and $A_{j} \cap A_{j+1}=\emptyset$ for all $j \in N$, and

(2) for each $j \in N$ the set $\left\{x \in \mathbf{R}^{n}\left|s r_{j} \leq\right| x \mid \leq r_{j+1}\right\} \cap E$ contains at most $p-2(p=p(n, K))$ points.

If $W=\left\{b_{1}, \ldots, b_{p-1}\right\} \subset \mathbf{R}^{n}$ consists of $p-1$ different points and $f^{-1} W \subset E$, then the $\operatorname{limit}_{\lim } \rightarrow \infty f(x)$ exists.

Proof. We may assume that $f$ is nonconstant. Let $\varphi_{j}$ denote the map $x \mapsto r_{j} x / r_{1}, x \in A_{1}$. By the generalized Montel's theorem, the family $\{f \circ$ $\left.\varphi_{j} \mid j \in N\right\}$ is normal in $A_{1}$. Let $\mathcal{F}$ stand for the cluster set of the sequence $\left(f \circ \varphi_{j}\right)$, i.e., $\mathcal{F}$ consists of all mappings which occur as limits of various subsequences of $\left(f \circ \varphi_{j}\right)$ (the topology is of course that of uniform convergence on compact subsets). If $\mathcal{F}$ contains a quasiregular mapping, then $f$ is bounded in the neighborhood of $\infty$. This implies that $\infty$ is a removable singularity, i.e., $\lim _{x \rightarrow \infty} f(x)$ exists. If $\mathcal{F}$ contains only the constant map $x \mapsto \infty$, then $f\left(\varphi_{j}(x)\right) \rightarrow \infty$ uniformly in $S^{n-1}\left(s^{1 / 2} r_{1}\right)$. Hence there is $j^{\prime} \in N$ such that $|f(x)|>M=\max \left\{\left|b_{1}\right|, \ldots,\left|b_{p-1}\right|\right\}$ for $x \in S^{n-1}\left(s^{1 / 2} r_{j}\right)$ whenever $j \geq j^{\prime}$. Consider the closed annulus $C_{j}=\bar{B}^{n}\left(s^{1 / 2} r_{j+1}\right) \backslash B^{n}\left(s^{1 / 2} r_{j}\right), j \geq j^{\prime}$. It follows from the hypothesis of the theorem that $C_{j} \cap E$ contains at most $p-2$ points. Therefore $B^{n}(M) \not \subset f C_{j}$. Making use of the relation $f \partial C_{j} \subset \mathbf{R}^{n} \backslash \bar{B}^{n}(M)$ and the openness of $f$, we infer that $f C_{j} \subset \mathbf{R}^{n} \backslash \bar{B}^{n}(M)$. Now the minimum principle implies that $\lim _{x \rightarrow \infty} f(x)=\infty$. 口

Let $D$ be a proper subdomain of $\mathbf{R}^{n}$. Then $D$ can be equipped with the quasihyperbolic metric $k_{D}(\cdot, \cdot)$. Let $f: D \rightarrow \overline{\mathbf{R}}^{n}$ be a quasimeromorphic mapping. Following Vuorinen [13, p. 163] we say that $f$ is normal provided that it is uniformly continuous with respect to $k_{D}(\cdot, \cdot)$ (the range space $\overline{\mathbf{R}}^{n}$ is equipped with the chordal metric). It must be pointed out that normality in the sense of Lehto and Virtanen (in dimension 2) is equivalent to uniform continuity with respect to the hyperbolic metric; see e.g. [4]. We first show that isolated singularities are nonessential for normal quasimeromorphic mapping omitting at least one point.

Theorem 2. Let $R>0$ and let $f: \mathbf{R}^{n} \backslash \bar{B}^{n}(R) \rightarrow \mathbf{R}^{n}$ be a normal quasiregular mapping. Then the limit $\lim _{x \rightarrow \infty} f(x)$ exists. 
Proof. Let $r>1$ and let $\varphi_{r}$ stand for the map $x \mapsto r x, x \in D=\mathbf{R}^{n} \backslash \bar{B}^{n}(R)$. Since $\varphi_{r}$ is distance-decreasing, i.e., $k_{D}\left(\varphi_{r}(x), \varphi_{r}(y)\right) \leq k_{D}(x, y)$ for all $x, y \in D$, the family $\left\{f \circ \varphi_{r} \mid r>1\right\}$ is equicontinuous in $D$. By the Arzela-Ascoli theorem, $\left\{f \circ \varphi_{r} \mid r>1\right\}$ is a normal family. Consider the sequence $\left(f \circ \varphi_{2^{j}}\right)$ restricted to $B^{n}(8 R) \backslash \bar{B}^{n}(R)$. Let $\mathcal{F}$ again denote the cluster set of this sequence. If $\mathcal{F}$ contains a quasiregular mapping, $f$ is bounded in a neighborhood of $\infty$, and

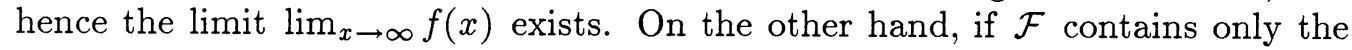
constant map $x \mapsto \infty$, then the number of the zeros of $f$ must be finite. Indeed, if $a_{1}, a_{2}, \ldots$ are the zeros of $f$, we can choose the natural numbers $j_{1}, j_{2}, \ldots$ so that $a_{i} / 2^{j_{i}} \in \bar{B}^{n}(4 R) \backslash B^{n}(2 R)$; then $\left(f \circ \varphi_{2^{j_{i}}}\right)$ contains a subsequence, whose limit mapping attains the value 0 . Accordingly, we may apply the minimum principle to obtain $\lim _{x \rightarrow \infty} f(x)=\infty$.

Remark. Theorem 2 does not hold for normal quasimeromophic mappings. For instance, the meromorphic function

$$
f: f(z)=\frac{\prod_{1}^{\infty}\left(1-z 2^{-i}\right)}{\prod_{1}^{\infty}\left(1+z 2^{-i}\right)}
$$

fulfils the condition $\lim _{z \rightarrow \infty} \sup |z| f^{*}(z)<\infty$, where $f^{*}(z)$ stands for $\left|f^{\prime}(z)\right| /(1+$ $\left.|f(z)|^{2}\right)$. This means that $f$ is normal in any domain of the form $\{z \in \mathbf{C}|| z \mid>$ $R\}$. See Section 4 for further information concerning the expression $|z| f^{*}(z)$.

Recalling that the omitted values are asymptotic values at an essential isolated singularity [7, p. 16], the next result can be regarded as a generalization of the preceding theorem. The proof is a fairly standard normal family argument and is omitted.

Theorem 3. Let $R>0$ and let $f: \mathbf{R}^{n} \backslash \bar{B}^{n}(R) \rightarrow \overline{\mathbf{R}}^{n}$ be a normal quasimeromorphic mapping possessing an asymptotic value at $\infty$. Then the limit $\lim _{x \rightarrow \infty} f(x)$ exists.

In the special case of meromorphic functions the validity of Theorems 2 and 3 was observed already by Lehto and Virtanen [4, pp. 7-8]. In fact, their concern there was the class of weakly normal functions, i.e., functions whose restrictions to every simply connected subdomain are normal with respect to the hyperbolic metric. However, by [4, Theorem 2] a function defined in a set of type $\{z \in$ $\left.\mathrm{C}|0<| z_{0}|\leq| z \mid<\infty\right\}$ is weakly normal if and only if $\lim _{z \rightarrow \infty} \sup |z| f^{*}(z)<\infty$. Furthermore, we are going to show that this happens exactly when $f$ is normal with respect to the quasihyperbolic metric of $D=\left\{z \in \mathbf{C}|| z_{0}|<| z \mid<\infty\right\}$. Since $f$ is meromorphic also on $S^{1}\left(\left|z_{0}\right|\right), \lim _{z \rightarrow \infty} \sup |z| f^{*}<\infty$ if and only if $\sup \left\{\left(|z|-\left|z_{0}\right|\right) f^{*}(z) \mid z \in D\right\}<\infty$. It is clear that the latter relation implies the uniform continuity of $f$ with respect to $k_{D}$. Now assume that $f$ is uniformly continuous with respect to $k_{D}$ and choose $\delta>0$ such that $q\left(f(z), f\left(z^{\prime}\right)\right)<\frac{1}{2}$ 
whenever $k_{D}\left(z, z^{\prime}\right)<\delta$. Fix $z^{\prime} \in D$. Since the spherical derivative is invariant under rotations of the sphere, we may assume that $f\left(z^{\prime}\right)=0$. Set $r=\left(\left|z^{\prime}\right|-\right.$ $\left.\left|z_{0}\right|\right)\left(1-e^{-\delta}\right)$. A simple calculation shows that

$$
B^{2}\left(z^{\prime}, r\right) \subset\left\{z \in \mathbf{C} \mid k_{D}\left(z^{\prime}, z\right)<\delta\right\} .
$$

Observe that $|f(z)|<1$ for $z \in B^{2}\left(z^{\prime}, r\right)$. Hence by Schwarz's lemma, $f^{*}\left(z^{\prime}\right)=$ $\left|f^{\prime}\left(z^{\prime}\right)\right|<r^{-1}$, whence $\left(\left|z^{\prime}\right|-\left|z_{0}\right|\right) f^{*}\left(z^{\prime}\right)<\left(1-e^{-\delta}\right)^{-1}$. Thus

$$
\sup \left\{\left(|z|-\left|z_{0}\right|\right) f^{*}(z) \mid z \in D\right\}<\infty .
$$

The next theorem shows that the equivalence of the two concepts generally prevails.

Theorem 4. Let $D$ be a proper subdomain of $\mathbf{C}$, and let $f: D \rightarrow \hat{\mathbf{C}}=$ $\mathbf{C} \cup\{\infty\}$ be a quasimeromorphic mapping. Then $f$ is weakly normal if and only if $f$ is normal with respect to the quasihyperbolic metric of $D$.

Proof. First suppose that $f$ is normal with respect to the quasihyperbolic metric. Let $D^{\prime}$ be an arbitrary simply connected subdomain of $D$, and fix $z \in D^{\prime}$. It is well-known, and easy to prove by means of Koebe's Viertelsatz, that $\varrho(z)$, the hyperbolic density of $D^{\prime}$ at $z$ satisfies $\varrho(z) \geq \frac{1}{4} d\left(z, \partial D^{\prime}\right)^{-1}$. Therefore

$$
\begin{aligned}
\varrho(z) \geq & \frac{1}{4} \frac{1}{d\left(z, \partial D^{\prime}\right)} \geq \frac{1}{4} \frac{1}{d(z, \partial D)} \\
& =\frac{1}{4} \text { the quasihyperbolic density at } z .
\end{aligned}
$$

Hence $f$ is normal in $D^{\prime}$ in the sense of Lehto-Virtanen. This means that $f$ is weakly normal.

Now assume that $f$ is weakly normal in $D$. Suppose for the moment that $f$ is not normal with respect to $k_{D}$. Then there is an $\varepsilon>0$ and two sequences $\left(z_{j}\right)$ and $\left(z_{j}^{\prime}\right)$ of points in $D$ such that $k_{D}\left(z_{j}, z_{j}^{\prime}\right) \rightarrow 0$ and $q\left(f\left(z_{j}\right), f\left(z_{j}^{\prime}\right)\right) \geq \varepsilon$. Set $r_{j}=$ $d\left(z_{j}, \partial D\right), j \in N$. By passing to a subsequence if necessary we may assume that $B^{2}\left(z_{i}, r_{i}\right) \cap B^{2}\left(z_{j}, r_{j}\right)=\emptyset$ for $i \neq j$ and $z_{j}^{\prime} \in B^{2}\left(z_{j}, \frac{1}{2} r_{j}\right)$ for each $j$. Obviously, given any $j \in N$ we can connect $B^{2}\left(z_{j}, \frac{1}{2} r_{j}\right)$ and $B^{2}\left(z_{j+1}, \frac{1}{2} r_{j+1}\right)$ with a narrow corridor $C_{j} \subset D \backslash \cup_{i=1}^{\infty} B^{2}\left(z_{i}, \frac{1}{2} r_{i}\right)$ such that the set $D^{\prime}=\cup_{i=1}^{\infty}\left(B^{2}\left(z_{i}, \frac{1}{2} r_{i}\right) \cup C_{i}\right)$ is a simply connected subdomain of $D$. We are going to show that $f \mid D^{\prime}$ is not normal with respect to $\delta(\cdot, \cdot)$, the hyperbolic metric of $D^{\prime}$. First, $\lim _{j \rightarrow \infty} k_{D}\left(z_{j}, z_{j}^{\prime}\right)=0$ implies that $d\left(z_{j}, z_{j}^{\prime}\right) / r_{j} \rightarrow 0$ as $j \rightarrow \infty$. On the other hand, $\varrho(z)$, the density of $\delta(\cdot, \cdot)$ at $z \in D^{\prime}$, satisfies $\varrho(z) \leq d\left(z, \partial D^{\prime}\right)^{-1}$. In particular $\varrho\left(z_{j}\right) \leq 2 / r_{j}$ for each $j$. It follows that $\delta\left(z_{j}, z_{j}^{\prime}\right) \rightarrow 0$ as $j \rightarrow \infty$. Yet $q\left(f\left(z_{j}\right), f\left(z_{j}^{\prime}\right)\right) \geq \varepsilon$. Thus $f \mid D^{\prime}$ fails to be uniformly continuous with respect to $\delta(\cdot, \cdot)$. This contradiction with the assumption completes the proof. $\square$ 
Remark. Other characterizations of normality with respect to the quasihyperbolic metric in dimension 2 can be found in the recent work of Minda [8].

We conclude this section with another result on Picard sets, which is modelled on a theorem by Toppila [12, Theorem 3].

Theorem 5. Let $R>0$ and let $f: D=\mathbf{R}^{n} \backslash \bar{B}^{n}(R) \rightarrow \mathbf{R}^{n}$ be a $K$ quasiregular mapping. Let $E=\left\{a_{j} \mid j \in N\right\}$ be a subset of $D$ with $a_{j} \rightarrow \infty$ as $j \rightarrow \infty$ such that there is an $\varepsilon>0$ with $B^{n}\left(a_{j}, \varepsilon\left|a_{j}\right|\right) \subset D$ and $B^{n}\left(a_{j}, \varepsilon\left|a_{j}\right|\right) \cap E=$ $\left\{a_{j}\right\}$ for large $j$. If $W=\left\{b_{1}, \ldots, b_{p-1}\right\} \subset \mathbf{R}^{n}$ consists of $p-1$ different points $(p=p(n, K))$ and $f^{-1} W \subset E$, then the limit $\lim _{x \rightarrow \infty} f(x)$ exists.

Proof. Suppose that $\infty$ is an essential singularity for $f$. It then follows from Theorem 2 that $f$ cannot be normal in $D$. Thus there is $c>0$ and two sequences $\left(x_{j}\right)$ and $\left(y_{j}\right)$ of points of $D$ such that $x_{j} \rightarrow \infty, d\left(x_{j}, y_{j}\right) /\left(\left|x_{j}\right|-R\right) \rightarrow 0$ and $q\left(f\left(x_{j}\right), f\left(y_{j}\right)\right) \geq c$. Set $B_{j}=B^{n}\left(x_{j}, \frac{1}{2} \varepsilon\left|x_{j}\right|\right)$; we may assume that $B_{j} \subset D$ and $y_{j} \in B^{n}\left(x_{j}, \frac{1}{8} \varepsilon\left|x_{j}\right|\right)$ for each $j \in N$. Let $\varphi_{j}$ denote a similarity transformation of $B_{1}$ onto $B_{j}$ and set $y_{j}^{\prime}=\varphi_{j}^{-1}\left(y_{j}\right)$. Then

$$
d\left(x_{1}, y_{j}^{\prime}\right)=\frac{d\left(x_{j}, y_{j}\right)}{\left|x_{j}\right|}\left|x_{1}\right|=\frac{d\left(x_{j}, y_{j}\right)}{\left|x_{j}\right|-R} \frac{\left|x_{j}\right|-R}{\left|x_{j}\right|}\left|x_{1}\right| \rightarrow 0
$$

and

$$
q\left(f\left(\varphi_{j}\left(x_{1}\right)\right), f\left(\varphi_{j}\left(y_{j}^{\prime}\right)\right)\right)=q\left(f\left(x_{j}\right), f\left(y_{j}\right)\right) \geq c .
$$

Thus the family $\left\{f \circ \varphi_{j} \mid j \in N\right\}$ is not normal in $B^{n}\left(x_{1}, \frac{1}{8} \varepsilon\left|x_{1}\right|\right)$. The same is of course true for each family consisting of some subsequence of $\left(f \circ \varphi_{j}\right)$. Hence by the generalized Montel's theorem (see Section 2) $f \circ \varphi_{j}$ assumes in $B^{n}\left(x_{1}, \frac{1}{8} \varepsilon\left|x_{1}\right|\right)$ at least one of the values $b_{1}, \ldots, b_{p-1}$ for large $j$. By passing to a subsequence and relabeling we may assume that $f \circ \varphi_{j}$ attains $b_{1}$ for each $j \in N$. On the other hand, it follows from the hypothesis of the theorem that for large $i, B_{j}$ contains at most one point of $E$. Therefore the image of the annulus $B^{n}\left(x_{j}, \frac{1}{2} \varepsilon\left|x_{j}\right|\right) \backslash \bar{B}^{n}\left(x_{j}, \frac{1}{8} \varepsilon\left|x_{j}\right|\right)$ under $f$ does not meet $W$ for large $j$. Furthermore, $b_{1}$ and $b_{2}$ do not belong to the same component of $\mathbf{R}^{n} \backslash f S^{n-1}\left(x_{j}, \frac{1}{4} \varepsilon\left|x_{j}\right|\right)$ for large $j$ (see [13, p. 121]). Thus we can find an $M>0$ such that

$$
\min \left\{|f(x)| \mid x \in S^{n-1}\left(x_{j}, \frac{1}{4} \varepsilon\left|x_{j}\right|\right)\right\}<M
$$

for all $j \in N$. Invoking the generalized Schottky theorem $[13$, p. 166] we see that $f$ is bounded in $\cup_{j=1}^{\infty} S^{n-1}\left(x_{j}, \frac{1}{4} \varepsilon\left|x_{j}\right|\right)$, whereas the maximum principle implies that $f$ is bounded in $\cup_{j=1}^{\infty} B^{n}\left(x_{j}, \frac{1}{4} \varepsilon\left|x_{j}\right|\right)$. But this state of affairs contradicts the fact that $\left\{f \circ \varphi_{j}\right\}$ (or any of its subfamilies) is not normal in $B^{n}\left(x_{1}, \frac{1}{8} \varepsilon\left|x_{1}\right|\right)$. व

Remark. Both Theorem 1 and Theorem 5 generalize a recent result by Vuorinen [14, Theorem 1.1]. 
3. In this section we make some observations on the value distribution of normal quasimeromorphic mappings in the neighborhood of an isolated singularity. For the sake of convenience we assume that the mappings are defined in the whole of $\mathbf{R}^{n}$. Theorem 6 below partly generalizes the work of A. Ostrowski on exceptional meromorphic functions in the sense of Julia [10, pp. 245-249]. Recall that $f$, a function meromorphic in the complex plane, is said to be exceptional provided that for any sequence $\left(\sigma_{j}\right)$ of complex numbers with $\lim _{j \rightarrow \infty}=\infty$ the family $\left\{z \mapsto f\left(\sigma_{j} z\right) \mid j \in N\right\}$ is normal in $\mathbf{C} \backslash\{0\}$. The equivalence of the two notions in question is established in the following lemma; cf. [4, Theorem 3]. The proof is straightforward and hence omitted.

Lemma 1. Let $f: \mathbf{R}^{n} \rightarrow \overline{\mathbf{R}}^{n}$ be a quasimeromorphic mapping. Then the following statements are equivalent:

(1) $f \mid \mathbf{R}^{n} \backslash\{0\}$ is normal.

(2) Let $\mathcal{S}_{0}$ stand for the group of similarity transformations of $\mathbf{R}^{n}$ fixing the origin. Then the family $\left\{f \circ \varphi \mid \varphi \in \mathcal{S}_{0}\right\}$ is normal in $\mathbf{R}^{n} \backslash\{0\}$.

(3) Let $\left(r_{j}\right)$ be a sequence of positive numbers with $r_{j} \rightarrow \infty$. Then the family $\left\{x \mapsto f\left(r_{j} x\right) \mid j \in N\right\}$ is normal in $\mathbf{R}^{n} \backslash\{0\}$.

(4) The family $\left\{x \mapsto f\left(2^{j} x\right) \mid j \in N\right\}$ is normal in the annulus $B^{n}(4) \backslash \bar{B}^{n}\left(\frac{1}{2}\right)$.

Let $f: \mathbf{R}^{n} \rightarrow \overline{\mathbf{R}}^{n}$ be quasimeromorphic and let $r>0$. Given $y \in \overline{\mathbf{R}}^{n}$ we set

$$
n_{f}(r, y)=\sum_{j=1}^{k} i\left(x_{j}, f\right) ;
$$

here $\left\{x_{1}, \ldots, x_{k}\right\}=f^{-1}(y) \cap \bar{B}^{n}(r)$ and $i\left(x_{j}, f\right)$ is the local index of $f$ at $x_{j}$ (see $[13$, p. 123]). We also set

$$
\bar{n}_{f}(r, y)=\sum_{j=1}^{m} i\left(z_{j}, f\right),
$$

where $\left\{z_{1}, \ldots, z_{m}\right\}=f^{-1}(y) \cap\left(\bar{B}^{n}(2 r) \backslash B^{n}(r)\right)$. In order to obtain estimates for the differences of the counting function we shall make use of the notion of the winding number. This is defined as follows. Let $S$ be an $(n-1)$-sphere in $\mathbf{R}^{n}$, let $f: S \rightarrow \mathbf{R}^{n}$ be a continuous map and let $y \in \mathbf{R}^{n} \backslash f S$. Consider the map $u: S \rightarrow S^{n-1}$

$$
u(x)=\frac{f(x)-y}{|f(x)-y|} .
$$

Then the winding number of $f$ with respect to $y$ is defined as

$$
w(f, y)=\operatorname{deg}(u),
$$

where deg refers to the topological degree. If then $S=\cup_{i=1}^{k} S_{i}$ is a finite union of mutually disjoint spheres $S_{i}, w(f, y)$ stands for $\sum_{i=1}^{k} w\left(f \mid S_{i}, y\right)$. Observe that 
$w(f, y)$ depends on the orientation of the spheres. In what follows, the spheres always constitute a part of the boundary of a relatively compact subdomain of $\mathbf{R}^{n}$, and it is understood that the orientation is positive with respect to this domain.

Lemma 2. Let $r>0$ and let $f$ be a nonconstant quasimeromorphic mapping defined in a neighborhood of $\bar{B}^{n}(r)$. Suppose that $f S^{n-1}(r) \cap\{0, \infty\}=\emptyset$. Then $w\left(f \mid S^{n-1}(r), 0\right)=n_{f}(r, 0)-n_{f}(r, \infty)$.

Proof. Let $f^{-1}(\infty) \cap B^{n}(r)=\left\{x_{1}, \ldots, x_{k}\right\}$. For each $j \in\{1, \ldots, k\}$ choose $r_{j}>0$ such that $\bar{B}^{n}\left(x_{j}, r_{j}\right) \subset B^{n}(r), f \bar{B}^{n}\left(x_{j}, r_{j}\right) \cap \bar{B}^{n}=\emptyset$ and $\bar{B}^{n}\left(x_{i}, r_{i}\right) \cap$ $\bar{B}^{n}\left(x_{j}, r_{j}\right)=\emptyset$ for $i \neq j$. Set $D=B^{n}(r) \backslash \bar{B}^{n}\left(x_{j}, r_{j}\right)$. It follows from the definition of the local index and [1, Kap. XII, Sektion 2, Satz II] (see also [1, Kap. XII, Sektion 1, Nr. 5]) that

$$
i\left(x_{j}, f\right)=-w\left(f \mid \partial B^{n}\left(x_{j}, r_{j}\right), 0\right) \quad j=1, \ldots, k,
$$

(observe that reversing the orientation changes the sign of the degree). By the same Satz

$$
\begin{aligned}
n_{f}(r, 0) & =w(f \mid \partial D, 0)=w\left(f \mid \partial B^{n}(r), 0\right)+w\left(f \mid \partial D \backslash \partial B^{n}(r), 0\right) \\
& =w\left(f \mid S^{n-1}(r), 0\right)-\sum_{j=1}^{k} w\left(f \mid \partial B^{n}\left(x_{j}, r_{j}\right), 0\right) \\
& =w\left(f \mid S^{n-1}(r), 0\right)+\sum_{j=1}^{k} i\left(x_{j}, f\right) \\
& =w\left(f \mid S^{n-1}(r), 0\right)+n_{f}(r, \infty) .
\end{aligned}
$$

The assertion follows. $\square$

Theorem 6. Let $f: \mathbf{R}^{n} \rightarrow \overline{\mathbf{R}}^{n}$ be a quasimeromorphic mapping such that $f \mid \mathbf{R}^{n} \backslash\{0\}$ is normal. Then:

(1) For each $d>0$ there is a positive constant $C_{1}=C_{1}(d, f)$, depending only on $d$ and $f$, such that given any two points $a, b \in \overline{\mathbf{R}}^{n}$ with $q(a, b) \geq d$, then $\left|n_{f}(r, a)-n_{f}(r, b)\right| \leq C_{1}$ for all $r>0$.

(2) There is a positive constant $C_{2}=C_{2}(f)$, depending only on $f$, such that $\bar{n}_{f}(r, a) \leq C_{2}$ for all $r>0$ and all $a \in \overline{\mathbf{R}}^{n}$.

(3) Let $a$ and $b$ be two distinct points of $\overline{\mathbf{R}}^{n}$, and let $f^{-1}(a)=\left\{a_{j} \mid j \in N\right\}$, $f^{-1}(b)=\left\{b_{j} \mid j \in N\right\}$. Then

$$
\inf \left\{\frac{\left|a_{j}-b_{j}\right|}{\left|a_{i}\right|} \mid i, j \in N\right\}>0 .
$$

Proof. We may assume that $\infty$ is an essential singularity for $f$. By Theorem $2, f$ then assumes all values of $\overline{\mathbf{R}}^{n}$ infinitely often. 
To prove (1) we fix $d>0$ and suppose there are two sequences $\left(a_{j}\right)$ and $\left(b_{j}\right)$ of points of $\overline{\mathbf{R}}^{n}$ and a sequence of positive numbers $\left(r_{j}\right)$ such that $q\left(a_{j}, b_{j}\right) \geq d$ and $\left|n_{f}\left(r_{j}, a_{j}\right)-n_{f}\left(r_{j}, b_{j}\right)\right| \rightarrow \infty$ as $j \rightarrow \infty$. By passing to subsequences we may assume that there are two points $a, b \in \overline{\mathbf{R}}^{n}$ such that $a_{j} \rightarrow a, b_{j} \rightarrow b$ and $q(a, b) \geq d$. Let $\psi$ be a Möbius transformation of $\overline{\mathbf{R}}^{n}$ sending $a$ and $b$ to 0 and $\infty$, respectively, and set $g=\psi \circ f$. We may assume that $\left|\psi\left(a_{j}\right)\right|<1$ and $\left|\psi\left(b_{j}\right)\right|>1$ for all $j$. We next modify $\left(r_{j}\right)$ in the manner indicated by Ostrowski $[10$, p. 246]. Fix $j \in N$, and assume first that

$$
n_{f}\left(r_{j}, a_{j}\right)=n_{g}\left(r_{j}, \psi\left(a_{j}\right)\right) \geq n_{f}\left(r_{j}, b_{j}\right)=n_{g}\left(r_{j}, \psi\left(b_{j}\right)\right) .
$$

Set $r_{j}^{\prime}=\max \left\{|x| \mid x \in \bar{B}^{n}\left(r_{j}\right)\right.$ and $\left.g(x)=\psi\left(a_{j}\right)\right\}, r_{j}^{\prime \prime}=\min \left\{|x| \mid x \in \mathbf{R}^{n} \backslash\right.$ $B^{n}\left(r_{j}\right)$ and $\left.g(x)=\psi\left(b_{j}\right)\right\}$. By continuity, there is $r_{j}^{\prime \prime \prime} \in\left[r_{j}^{\prime}, r_{j}^{\prime \prime}\right]$ such that $g S^{n-1}\left(r_{j}^{\prime \prime \prime}\right) \cap S^{n-1} \neq \emptyset$. Furthermore,

$$
\left|n_{g}\left(r_{j}^{\prime \prime \prime}, \psi\left(a_{j}\right)\right)-n_{g}\left(r_{j}^{\prime \prime \prime}, \psi\left(b_{j}\right)\right)\right| \geq\left|n_{g}\left(r_{j}, \psi\left(a_{j}\right)\right)-n_{g}\left(r_{j}, \psi\left(b_{j}\right)\right)\right| .
$$

Similarly, if $n_{g}\left(r_{j}, \psi\left(a_{j}\right)\right)<n_{g}\left(r_{j}, \psi\left(b_{j}\right)\right)$ we can find $r_{j}^{\prime \prime \prime}$ such that $g S^{n-1}\left(r_{j}^{\prime \prime \prime}\right) \cap$ $S^{n} \neq \emptyset$ and

$$
n_{g}\left(r_{j}^{\prime \prime \prime}, \psi\left(b_{j}\right)\right)-n_{g}\left(r_{j}^{\prime \prime \prime}, \psi\left(a_{j}\right)\right) \geq n_{g}\left(r_{j}, \psi\left(b_{j}\right)\right)-n_{g}\left(r_{j}, \psi\left(a_{j}\right)\right) .
$$

We then replace $r_{j}$ by $r_{j}^{\prime \prime \prime}$ but retain the notation $\left(r_{j}\right)$ for the new sequence. Altogether, we have a sequence $\left(r_{j}\right)$ such that

$$
\text { and } \quad \begin{gathered}
\left|n_{g}\left(r_{j}, \psi\left(a_{j}\right)\right)-n_{g}\left(r_{j}, \psi\left(b_{j}\right)\right)\right| \rightarrow \infty \\
g S^{n-1}\left(r_{j}\right) \cap S^{n-1} \neq \emptyset \text { for all } j .
\end{gathered}
$$

Obviously $r_{j} \rightarrow \infty$ as $j \rightarrow \infty$.

Let $\varphi_{j}$ stand for the map $x \mapsto r_{j} x, j \in N$. By Lemma 1 , the family $\left\{g \circ \varphi_{j} \mid j \in N\right\}$ is normal in $\mathbf{R}^{n} \backslash\{0\}$. Hence, by passing to a subsequence once more, we may assume that $\left(g \circ \varphi_{j}\right)$ converges uniformly in the closed annulus $\bar{B}^{n}(2) \backslash B^{n}\left(\frac{1}{2}\right)$. By $(*) g \varphi_{j} S^{n-1} \cap S^{n-1} \neq \emptyset$. Therefore the limit mapping $h(x)=\lim g\left(\varphi_{j}(x)\right), x \in \bar{B}^{n}(2) \backslash B^{n}\left(\frac{1}{2}\right)$, is either a constant $c$ with $|c|=1$ or a nonconstant quasimeromorphic mapping. Assume first that $h(x) \equiv c$. Then $g \circ \varphi_{j} \mid S^{n-1}$ is homotopic to $h \mid S^{n-1}$ in $\mathbf{R}^{n} \backslash\{0\}$ for large $j$. Hence the same is true of the map

$$
x \mapsto \frac{g\left(\varphi_{j}(x)\right)}{\left|g\left(\varphi_{j}(x)\right)\right|}, \quad x \in S^{n-1},
$$


for large $j$. This implies that $w\left(g \circ \varphi_{j} \mid S^{n-1}, 0\right)=w\left(h \mid S^{n-1}, 0\right)=0$ for large $j$. Hence by Lemma $2 n_{g}\left(r_{j}, 0\right)=n_{g}\left(r_{j}, \infty\right)$ for large $j$. Furthermore, it follows from [13, 9.11 and 9.2.(1)] that

$$
n_{g}\left(r_{j}, \psi\left(a_{j}\right)\right)=n_{g}\left(r_{j}, 0\right)=n_{g}\left(r_{j}, \infty\right)=n_{g}\left(r_{j}, \psi\left(b_{j}\right)\right)
$$

for large $j$. But this contradicts $(*)$.

Suppose then that $h$ is nonconstant. It may happen that $h S^{n-1} \cap\{0, \infty\} \neq \emptyset$. However, we can find $R_{1} \in\left(\frac{1}{2}, 1\right)$ and $R_{2} \in(1,2)$ such that $h S^{n-1}\left(R_{1}\right) \cap\{0, \infty\}=$ $h S^{n-1}\left(R_{2}\right) \cap\{0, \infty\}=\emptyset$. Since $g \circ \varphi_{j} \rightarrow h$ uniformly in $S^{n-1}\left(R_{1}\right)$, we see as above that the maps $u: S^{n-1}\left(R_{1}\right) \rightarrow S^{n-1}$ and $v_{j}: S^{n-1}\left(R_{1}\right) \rightarrow S^{n-1}$

$$
u(x)=\frac{h(x)}{|h(x)|}, \quad v_{j}(x)=\frac{g\left(\varphi_{j}(x)\right)}{\left|g\left(\varphi_{j}(x)\right)\right|}
$$

are homotopic for large $j$. Hence

$$
w\left(h \mid S^{n-1}\left(R_{1}\right), 0\right)=w\left(g \circ \varphi_{j} \mid S^{n-1}\left(R_{1}\right), 0\right)
$$

for large $j$, whence by Lemma 2

$$
w\left(h \mid S^{n-1}\left(R_{1}\right), 0\right)=n_{g}\left(R_{1} r_{j}, 0\right)-n_{g}\left(R_{1} r_{j}, \infty\right) .
$$

Further by [13, 9.2(1)]

$$
n_{g}\left(R_{1} r_{j}, \psi\left(a_{j}\right)\right)=n_{g}\left(R_{1} r_{j}, 0\right)
$$

and

$$
n_{g}\left(R_{1} r_{j}, \psi\left(b_{j}\right)\right)=n_{g}\left(R_{1} r_{j}, \infty\right)
$$

for large $j$. Consequently, in order to arrive at a contradiction with $(*)$ it is enough to exhibit a positive $M$ such that for large $j$ the numbers of $\psi\left(a_{j}\right)$-points and $\psi\left(b_{j}\right)$-points of $g \circ \varphi_{j}$ in $\bar{B}^{n} \backslash B^{n}\left(R_{1}\right)$ (with due account of the local indices) are bounded by $M$. Since $g \circ \varphi_{j} \rightarrow h$ uniformly in $\bar{B}^{n}\left(R_{2}\right) \backslash B^{n}\left(R_{1}\right)$, we may apply [13, 9.11 and 9.2.(5)] to conclude that for large $j$

$$
\sum_{j=1}^{k} i\left(x_{j}, h\right)=n_{g \circ \varphi_{j}}\left(R_{2}, 0\right)-n_{g \circ \varphi_{j}}\left(R_{1}, 0\right)
$$

and

$$
\sum_{j=1}^{m} i\left(y_{j}, h\right)=n_{g \circ \varphi_{j}}\left(R_{2}, \infty\right)-n_{g \circ \varphi_{j}}\left(R_{1}, \infty\right)
$$


where $\left\{x_{1}, \ldots, x_{k}\right\}=h^{-1}(0) \cap\left(B^{n}\left(R_{2}\right) \backslash \bar{B}^{n}\left(R_{1}\right)\right)$ and $\left\{y_{1}, \ldots, y_{m}\right\}=h^{-1}(\infty) \cap$ $\left(B^{n}\left(R_{2}\right) \backslash \bar{B}^{n}\left(R_{1}\right)\right)$. On the other hand, it follows from [13, 9.11 and 9.2.(1)] that for large $j$

$$
n_{g \circ \varphi_{j}}\left(R_{2}, \psi\left(a_{j}\right)\right)-n_{g \circ \varphi_{j}}\left(R_{1}, \psi\left(a_{j}\right)\right)=n_{g \circ \varphi_{j}}\left(R_{2}, 0\right)-n_{g \circ \varphi_{j}}\left(R_{1}, 0\right)
$$

and

$$
n_{g \circ \varphi_{j}}\left(R_{2}, \psi\left(b_{j}\right)\right)-n_{g \circ \varphi_{j}}\left(R_{1}, \psi\left(b_{j}\right)\right)=n_{g \circ \varphi_{j}}\left(R_{2}, \infty\right)-n_{g \circ \varphi_{j}}\left(R_{1}, \infty\right) .
$$

Thus we may choose

$$
M=\max \left\{\sum_{j=1}^{k} i\left(x_{j}, h\right), \sum_{j=1}^{m} i\left(y_{j}, h\right)\right\} .
$$

The proof of (1) is thereby complete.

If (2) is not true, we can find a sequence $\left(a_{j}\right)$ of points of $\overline{\mathbf{R}}^{n}$ and a sequence $\left(r_{j}\right)$ of positive numbers tending to $\infty$ such that $\bar{n}_{f}\left(r_{j}, a_{j}\right) \rightarrow \infty$. By passing to subsequences we may assume that $a_{j} \rightarrow a$ for some $a \in \overline{\mathbf{R}}^{n}$ as $j \rightarrow \infty$. Let $\tilde{a} \in \overline{\mathbf{R}}^{n}$ denote the point antipodal to $a$, and let $\varphi_{j}$ be the map $x \mapsto r_{j} x, j \in N$. Then $\left\{f \circ \varphi_{j} \mid j \in N\right\}$ is normal in $\mathbf{R}^{n} \backslash\{0\}$. By passing to a subsequence we may assume that $\left(f \circ \varphi_{j}\right)$ converges uniformly in $\bar{B}^{n}(4) \backslash B^{n}\left(\frac{1}{2}\right)$. We infer from (1) that the limit mapping $g$ is not constant. Indeed, it follows from (1) that

$$
f^{-1}(\tilde{a}) \cap\left(\bar{B}^{n}\left(2 r_{j}\right) \backslash B^{n}\left(r_{j}\right)\right) \neq \emptyset
$$

for large $j$. Replacing $\bar{B}^{n}(2) \backslash B^{n}(1)$ by a slightly larger annulus $A$ with $a \notin g \partial A$ and arguing as in the proof of $(1)$, we see that $\bar{n}_{g}(1, a) \geq \bar{n}_{f \circ \varphi_{j}}\left(1, a_{j}\right)=\bar{n}_{f}\left(r_{j}, a_{j}\right)$ for large $j$. This contradiction completes the proof of (2).

The proof of (3) is rather trivial. If

$$
\inf \left\{\frac{\left|a_{i}-b_{j}\right|}{\left|a_{i}\right|} \mid i, j, \in N\right\}=0,
$$

we can find two subsequences $\left(a_{i_{m}}\right)$ and $\left(b_{j_{m}}\right)$ such that

$$
\frac{\left|a_{i_{m}}-b_{j_{m}}\right|}{\left|a_{i_{m}}\right|} \rightarrow 0, \quad \text { as } m \rightarrow \infty \text {. }
$$

Clearly this means that $k_{\mathbf{R}^{n} \backslash\{0\}}\left(a_{i_{m}}, b_{j_{m}}\right) \rightarrow 0$ as $m \rightarrow \infty$. Since

$$
q\left(f\left(a_{i_{m}}\right), f\left(b_{j_{m}}\right)\right)=q(a, b)>0,
$$

this state of affairs contradicts the uniform continuity of $f$. $\square$

Remark. With obvious modifications, Theorem 6 also holds for normal quasimeromorphic mappings defined in sets of type $\left\{x \in \mathbf{R}^{n}|| x \mid \geq M\right\}$. 
4. Let $f$ be a quasimeromorphic mapping having $\infty$ as an essential isolated singularity. What can be said about the size of the set $f S^{n-1}(r)$ as $r \rightarrow \infty$ ? This is the problem we will consider in this section.

Let $K \subset \overline{\mathbf{R}}^{n}$. The chordal diameter of $K$ is defined to be

$$
q(K)=\sup \{q(x, y) \mid x, y \in K\} .
$$

Given a sphere $S=S^{n}(x, r)$ in $\mathbf{R}^{n+1}$, a subset $S^{\prime}$ of $S$ is said to be an $(n-1)$ subsphere of $S$ provided that $S^{\prime}=S \cap H$, where $H$ is an $n$-hyperplane of $\mathbf{R}^{n+1}$ with $x \in H$.

Theorem 7. Let $R>0$ and let $f: \mathbf{R}^{n} \backslash \bar{B}^{n}(R) \rightarrow \overline{\mathbf{R}}^{n}=S^{n}\left(\frac{1}{2} e_{n+1}, \frac{1}{2}\right)=S$ be a quasimeromorphic mapping having an essential singularity at $\infty$. Then there is a sequence $\left(r_{j}\right)$ of positive numbers with $r_{j} \rightarrow \infty$ such that for each $j$ $f S^{n-1}\left(r_{j}\right)$ is contained in no open hemisphere of $S$, i.e., $f S^{n-1}\left(r_{j}\right)$ meets every $(n-1)$-subsphere of $S$. In particular,

$$
q\left(f S^{n-1}\left(r_{j}\right)\right) \geq\left(\frac{n+2}{2 n+2}\right)^{1 / 2} \quad \text { for all } j \in N .
$$

Proof. Suppose that from a certain $r_{0}$ each $f S^{n-1}(r)$ is contained in some open hemisphere of $S$. Let $S_{1}^{n-1}$ stand for an $(n-1)$-subsphere of $S$ such that $f S^{n-1}\left(r_{0}\right) \cap S_{1}^{n-1}=\emptyset$. Let $m_{n-1}$ denote the standard measure of $S_{1}^{n-1}$, and set

$$
r_{1}=\inf \left\{r \mid r>r_{0} \quad \text { and } \quad m_{n-1}\left(f\left(B^{n}(r) \backslash \bar{B}^{n}\left(r_{0}\right)\right) \cap S_{1}^{n-1}\right) \geq \frac{1}{2} m_{n-1}\left(S_{1}^{n-1}\right)\right\} .
$$

Observe that the above set is nonempty by the generalized Picard theorem (even the more elementary [6, Theorem 4.4] would do). Next choose an $(n-1)$-subsphere $S_{2}^{n-1}$ of $S$ such that $S_{2}^{n-1} \neq S_{1}^{n-1}$ and $f S^{n-1}\left(r_{1}\right) \cap S_{2}^{n-1}=\emptyset$. Then $S_{2}^{n-1}$ divides $S_{1}^{n-1}$ into two open hemispheres $S_{11}^{n-1}$ and $S_{12}^{n-1}$. At least one of these, say $S_{11}^{n-1}$, fulfils the condition $S_{11}^{n-1} \cap f S^{n-1}\left(r_{1}\right)=\emptyset$. If follows from the openness of $f$ that

$$
\bar{S}_{11}^{n-1} \subset f\left(B^{n}\left(r_{1}\right) \backslash \bar{B}^{n}\left(r_{0}\right)\right)
$$

or

$$
\bar{S}_{11}^{n-1} \cap f\left(B^{n}\left(r_{1}\right) \backslash \bar{B}^{n}\left(r_{0}\right)\right)=\emptyset .
$$

In the former case we have by continuity that

$$
\bar{S}_{11}^{n-1} \subset f\left(B^{n}\left(r_{2}\right) \backslash \bar{B}^{n}\left(r_{0}\right)\right)
$$

for some $r_{2}<r_{1}$, in contradiction to the choice of $r_{1}$. In the latter case

$$
\bar{S}_{11}^{n-1} \cap f\left(B^{n}\left(r_{3}\right) \backslash \bar{B}^{n}\left(r_{0}\right)\right)=\emptyset,
$$


for some $r_{3}>r_{1}$, again in contradiction to the choice of $r_{1}$.

Now let $\left(r_{j}\right)$ be a sequence described in the theorem and fix $j \in N$. We claim that $\bar{B}^{n+1}\left(\frac{1}{2} e_{n+1}, \frac{1}{2}\right)$ is the smallest closed ball in $\mathbf{R}^{n+1}$ containing $f S^{n-1}\left(r_{j}\right)$. Indeed, if $\bar{B}^{n+1}(x, r)$ is a closed ball with $r<\frac{1}{2}$, then $\bar{B}^{n+1}(x, r) \cap S$ is contained in an open hemisphere of $S$. Hence $f S^{n-1}\left(r_{j}\right) \not \subset \bar{B}^{n+1}(x, r)$. Jung's theorem [2, p. 357] now implies that $q\left(f S^{n-1}\left(r_{j}\right)\right) \geq((n+2) /(2 n+2))^{1 / 2}$ as was asserted. $\square$

Remark. We do not know whether the constant $((n+2) /(2 n+2))^{1 / 2}$ in the preceding theorem is the best possible. It seems feasible that it can be replaced even by 1 , i.e., that there is a sequence $\left(r_{j}\right)$ such that each sphere $S^{n-1}\left(r_{j}\right)$ contains two points which are mapped into antipodal points of $S$. In the case of meromorphic functions this is at least approximately true, for $\limsup _{r \rightarrow \infty} q\left(f S^{1}(r)\right)=1$ as appears from the proof of [5, Theorem 1]. Furthermore, there is a footnote in [5, p. 198] which claims that the same is true for planar quasimeromorphic mappings. However, we have not been able to verify this assertion.

The theorem of Lehto mentioned above ([5, Theorem 1]) asserts that

$$
\limsup _{z \rightarrow \infty}|z| f^{*}(z) \geq \frac{1}{2}
$$

and the constant $\frac{1}{2}$ is sharp. In fact, this statement can be expanded further as shown by

Theorem 8. Let $f$ be a meromorphic function defined in a neighborhood of the essential singularity $\infty$. Then there is a sequence $\left(z_{j}\right)$ tending to $\infty$ such that $\left|z_{j}\right| f^{*}\left(z_{j}\right) \geq \frac{1}{2}$ for each $j$.

Proof. Suppose there is $r_{0}>0$ such that $|z| f^{*}(z)<\frac{1}{2}$ for all $z$ with $|z| \geq r_{0}$. Then for all $r>r_{0}$ the spherical length of $f S^{1}(r)$ is less that $\pi$, the length of the great circles of the Riemann sphere $S$. It is known that such a curve is contained in an open hemisphere of $S$; see [3] for a very simple proof of this claim. But this is impossible by Theorem 7 . व

Corollary. Let $D$ be the open unit disc $\{z \in \mathbf{C}|| z \mid<1\}$ and let $f$ be a meromorphic function in $D^{*}=D \backslash\{0\}$ with $f^{*}(z)<\frac{1}{2}|z|^{-1}$ for $z \in D^{*}$. Then $f$ extends to a meromorphic function in $D$.

Added in proof. We have been informed that J. Heinonen and J. Rossi (to appear in Michigan Math. J.) have obtained results related to Theorem 7. In fact, their Theorem 2.3 combined with Remark 2.4 asserts in essence that, under the assumptions of Theorem $7, \lim \sup _{t \rightarrow \infty} q\left(f S^{n-1}(r)\right) \geq \frac{1}{4}$. Their method of proof is that of $[4$, Theorem 1$]$. 


\section{References}

[1] Alexandroff, P., and H. Hopf: Topologie. - Die Grundlehren der mathematischen Wissenschaften 45. Springer-Verlag, Berlin, 1935.

[2] Berger, M.: Geometry I. - Springer-Verlag, Berlin-Heidelberg-New York, 1987.

[3] Horn, R.A.: On Fenchel's theorem. - Amer. Math. Monthly 78, 1971, 380-381.

[4] Lehto, O., and K.I. Virtanen: On the behaviour of meromorphic functions in the neighborhood of an isolated singularity. - Ann. Acad. Sci. Fenn. Ser. A I Math. 240, 1957, 1-9.

[5] Lehto, O.: The spherical derivative of meromorphic functions in the neighborhood of an isolated singularity. - Comment. Math. Helv. 33, 1959, 196-205.

[6] Martio, O., S. Rickman, and J. VÄIsÄL̈̈: Distortion and singularities of quasiregular mappings. - Ann. Acad. Sci. Fenn. Ser. A I Math. 465, 1970, 1-13.

[7] Martio, O., S. Rickman, and J. VÄISÄLÄ: Topological and metric properties of quasiregular mappings. - Ann. Acad. Sci. Fenn. Ser. A I Math. 488, 1971, 1-31.

[8] Minda, D.: Bloch and normal functions on general planar regions. - MSRI Publications 10, edited by D. Drasin, C.J. Earle, F.W. Gehring, I. Kra and A. Marden. SpringerVerlag, Berlin-Heidelberg-New York, 1988, 101-110.

[9] Miniowitz, R.: Normal families of quasimeromorphic mappings. - Proc. Amer. Math. Soc. $84,1982,35-43$.

[10] Ostrowski, A.: Über Folgen analytischer Funktionen und einige Verschärfungen des Picardschen Satzes. - Math. Z. 24, 1925, 215-258.

[11] Rickman, S.: On the number of omitted values of entire quasiregular mappings. - J. Analyse Math. 37, 1980, 100-117.

[12] Toppila, S.: Some remarks on the value distribution of meromorphic functions. - Ark. Mat. 9, 1971, 1-9.

[13] Vuorinen, M.: Conformal geometry and quasiregular mappings. - Lecture Notes in Mathematics 1319. Springer-Verlag, Berlin-Heidelberg-New York, 1988.

[14] Vuorinen, M.: On Picard's theorem for entire quasiregular mappings. - Proc. Amer. Math. Soc. 107, 1989, 383-394.

University of Helsinki

Department of Mathematics

Hallituskatu 15

SF-00100 Helsinki

Finland

Received 23 October 1989 